\title{
A 2X2 MIMO Patch Antenna for Multi-Band Applications
}

\author{
Islam Md Rafiqul, Waheeb S. A.*, Sarah Rafiq, M. S. Yasmin and M. H. Habaebi \\ Department of Electrical and Computer Engineering, \\ Faculty of Engineering, International Islamic University Malaysia \\ Jalan Gombak 53100,Kuala Lumpur, Malaysia \\ e-mail: waheebtashan@yahoo.com
}

\begin{abstract}
In this paper, a Multiple Input Multiple Output (2x2MIMO) patch antenna for multiband applications has been proposed. It has been designed using groups of rings nearby the stepped cut at four corners of the patch and a separation in mid slot. This modification is proposed in order to increase the resonant frequencies and reduce the mutual coupling. The proposed 2x2 MIMO patch antenna is simulated using Computer Simulation Technology (CST), fabricated and tested. With such small geometrical dimensions, the proposed antenna is suitable for LTE (1.8 GHz), WiFi (2.4 GHz), and WiMax (3.5 GHz, $5.2 \mathrm{GHz}$ and $5.5 \mathrm{GHz}$ ) applications.
\end{abstract}

Keywords: Multiple Input Multiple Output (MIMO), Envelope correlation coefficient (ECC), Computer Simulation Technology (CST), Multi-Band Applications

\section{Introduction}

Multiple Input Multiple Output (MIMO) antennas are operating with multiple number of transmitters and receivers that are used to provide the high data rate required for services. The future wireless communication system is advancing towards MIMO to be used as the key technology. Therefore the utilization of MIMO has taken over many broadband applications. This is because the previous key technology such as Single Input Single Output (SISO) antenna encountered drawbacks such as multipath fading and low data rate capacity. On the other hand, MIMO is capable of achieving high speed data rate for both uplink and downlink channels, eliminate the issue of multipath fading, and can even achieve higher capacity without assigning more bandwidth. Therefore, this makes MIMO as one of the potential technologies. However, the antenna that interfaces with the wireless communication systems to the channel based on MIMO, is the most vulnerable component of the spatial degree of freedom [1-2]. In the design of traditional antenna, both noise and multipath affect the signal when it propagates from transmitter to receiver through various paths. In contrast to tradiational antenna design, modern antenna design employs multiple input multiple output, which enables the multipath effects to be utilized advantageously for the transmission of multiple data streams [1-3]. However, MIMO antenna's designs face challenges like the mutual coupling, which exists due to electromagnetic interaction between antenna elements, and therefore affects antenna efficiency. Furthermore, another major design challenge of MIMO antennas is the small spacing between antenna elements. The small spacing also leads to correlation between the antennas, hence reducing the channel capacity.

Patch antennas (also known as Microstrip antennas) are the most widely used antennas because of their low profile, small size, conformability, ease of use, lower cost, and versatility in terms of realization. Hence, these antennas are widely used in various useful applications [2-4]. In contrast rectangular microstrip patch antenna (RMPA) has few disadvantages such as low gain and narrow band. Most of the previous contributions in this research area were focused on increasing the bandwidth of RMPAs [5-6] by increasing the substrate thickness [2],[7]. In this paper, a $2 \times 2$ MIMO patch antenna has been proposed for multiband applications with groups of rings with the stepped cut at four corners and a mid slot separation, in order to increase resonant frequencies and decrease the mutual coupling. 


\section{Evaluation Process of Proposed Mimo Antenna Design Modes' Characteristics}

The structures of the patch antenna from single mode upto broadband are shown in Figure 1. The frequency ranges used in the design vary from $900 \mathrm{MHZ}$ to $3.5 \mathrm{GHz}$. Hence, this design can be used for various applications such as GSM (900 MHz/1.5 GHz), WiFi (2.4 GHz), LTE $(2.6 \mathrm{GHz})$ and WiMax $(3.5 \mathrm{GHz})$ applications. FR-4 substrate is applied in this design by a relative permittivity of $\boldsymbol{\varepsilon}_{\mathrm{r}}=4.3$. A thickness of the substrate $\mathbf{h}=1.6 \mathrm{~mm}$, Length $\mathbf{L s}=90 \mathrm{~mm}$ and width $\mathrm{fWS}=130 \mathrm{~mm}$ are used. The radiating patch has been designed on top of the dielectric substrate, while the ground plane is at the bottom. Radiating patch's material is of copper with a thickness of $t=0.035 \mathrm{~mm}$ and the conductivity of $\sigma=5.96 \mathrm{e} 7 \mathrm{~s} / \mathrm{m}$. The ground plane length is $\mathrm{LG}=18 \mathrm{~mm}$ and width is $\mathrm{WG}=90 \mathrm{~mm}$. The $50 \Omega$ output impedance matching was achieved by utilizing a feed transmission line with width of $W F=3 \mathrm{~mm}$ and length of $L F=20 \mathrm{~mm}$.

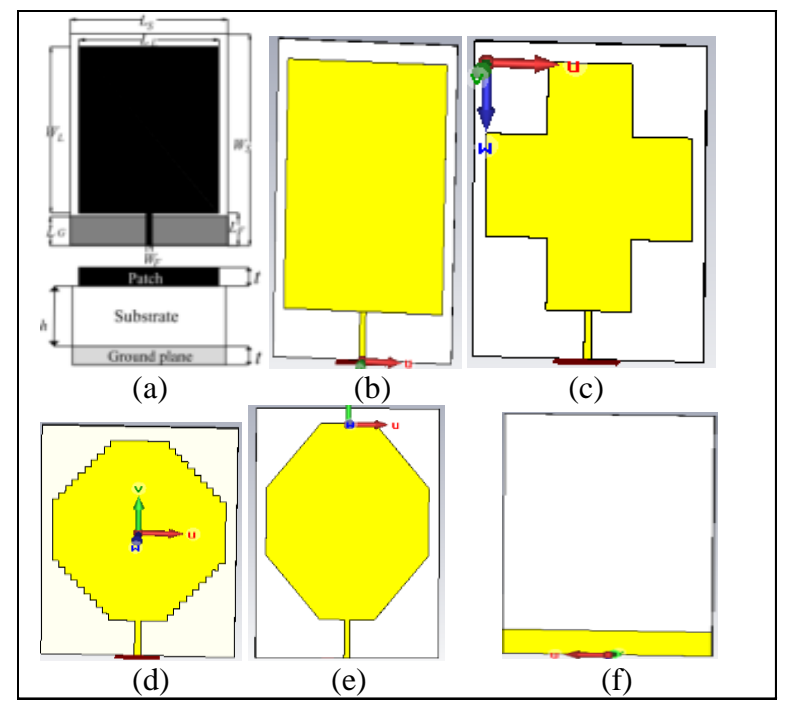

Figure 1. Designed structures of SISO antenna. (a)Top \& side view (b) Single mode RMPA (c) Dual mode modified RMPA (d) Multi-mode modified RMPA (e) Broadband modified RMPA (f) back view.

\section{2X2 MIMO ANTENNA DESIGN}

Figure 1(d) depicts the structure that has been used for MIMO antenna as shown in Figure 2 with the same specifications except for Ws=142 $\mathrm{mm}$ and separation in mid slot on the patch $D=10 \mathrm{~mm}$. Four ring's groups with FR-4 substrate were used, with each ring's group containing two rings, one as inner radius are $\operatorname{Rin}=3 \mathrm{~mm}$ and the other as outer radius Rout $=5$ $\mathrm{mm}$. To decrease the mutual coupling and improve the bandwidth of the proposed antenna, its final design makes use of mid slot separation and parasitic elements as shown in Figure 2. An improvement is shown in the simulation results of Figure 3 in terms of reflection coefficient and mutual coupling. It is obvious that frequency bands of $\operatorname{LTE}(1.8 \mathrm{GHz})$, Wifi(2.4) and WiMAX (3.5 $\mathrm{GHz}, 5.2 \mathrm{GHz}, 5.5 \mathrm{GHz}$ ) have been obtained.

To verify the simulation results, the proposed $2 \times 2$ MIMO antenna has been fabricated, tested and compared as shown in Figure 4. The rings that are presented in this design are crucial for the introduction of more new resonant frequencies. Beside that, mid space separation technique is employed to resolve the mutual coupling. The fabricated antenna has coupling coefficients below $-20 \mathrm{~dB}$ for the WiMAX $(3.5 \mathrm{GHz}, 5.2 \mathrm{GHz}$ and $5.5 \mathrm{GHz})$ bands and coupling coefficients of under $-15 \mathrm{~dB}$ for LTE $(1.8 \mathrm{GHz})$ and WiFi $(2.4 \mathrm{GHz})$ bands. The bandwidth in the fabricated design covers the range from $1.6 \mathrm{GHz}$ to $5.5 \mathrm{GHz}$ flat. 


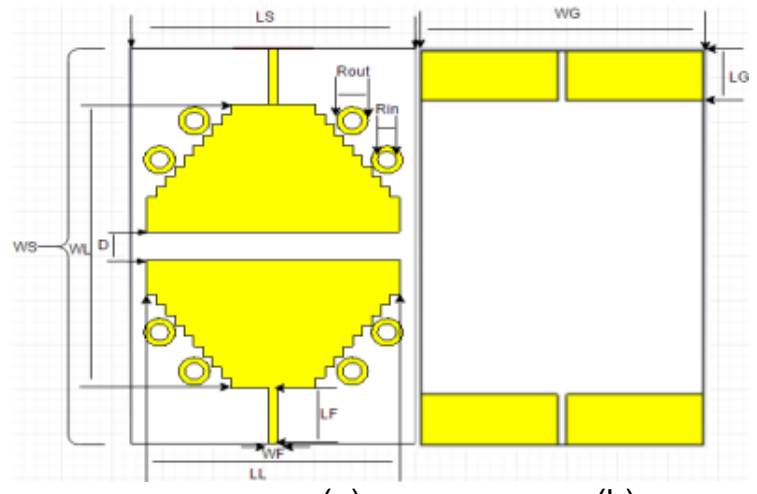

(a)

(b)

Figure 2. Proposed structure of 2x2 MIMO antenna system (a) Top view (b) back view

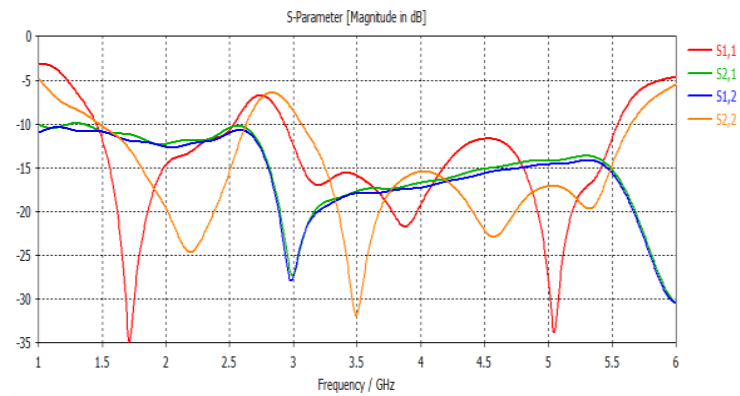

Figure 3. Simulated return loss and coupling coefficients for the proposed 2x2 MIMO antenna system.

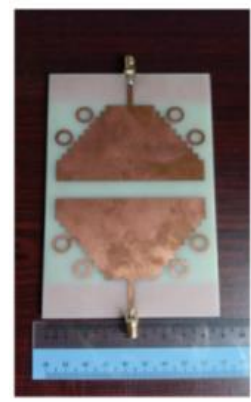

(a)

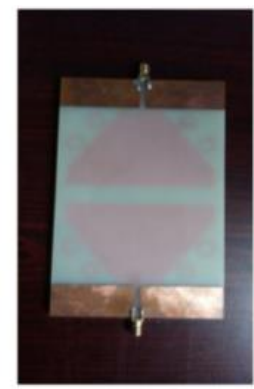

(b)

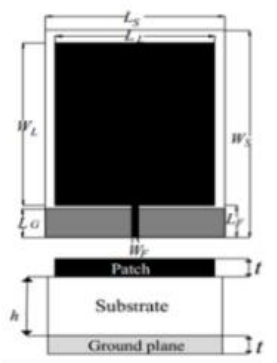

(c)

Figure 4. Fabricated 2x2 MIMO antenna system (a) Front view (b) top view (c) side view

Figure 5 shows the comparison of the measured and simulated return loss of $\mathrm{S} 11$ for the proposed antenna. Figure 5 shows that all the desired frequencies such as $1.8 \mathrm{GHz}, 2.4$ $\mathrm{GHz}, 3.5 \mathrm{GHz}, 5.2 \mathrm{GHz}$ and $5.5 \mathrm{GHz}$ have been obtained. 


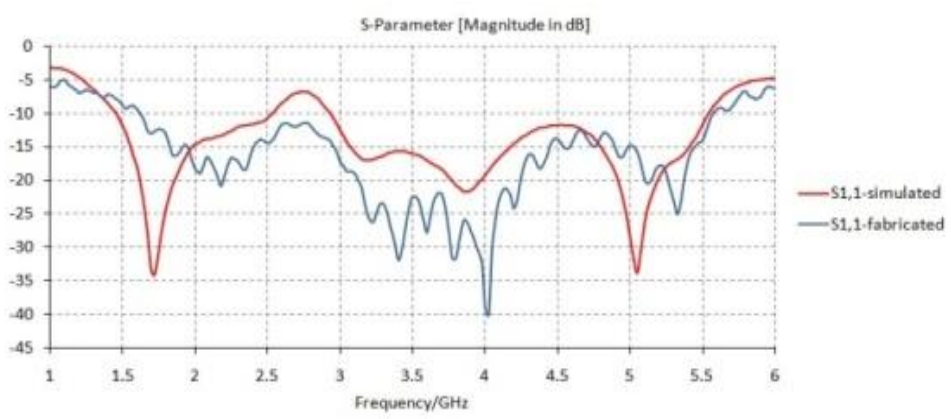

Figure 5. Simulated and measured S11 of the proposed 2x2 MIMO antenna system.

The comparison of the measured and simulated results of S22 for the proposed antenna is displayed in Figure 6. This Figure shows that all desired frequencies such as 1.8 $\mathrm{GHz}, 2.4 \mathrm{GHz}, 3.5 \mathrm{GHz}, 5.2 \mathrm{GHz}$ and $5.5 \mathrm{GHz}$ are also obtained.

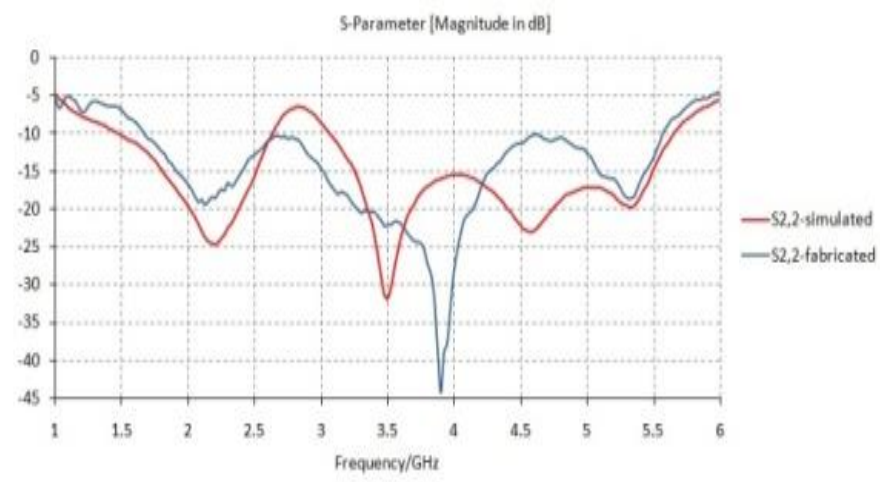

Figure 6. S22 simulated and measured of proposed 2x2 MIMO antenna system.

In order to have a good antenna array, the mutual coupling should be kept as low as possible. Spacing with $10 \mathrm{~mm}$ between the antenna elements and four groups of parasitic element rings,that have been distributed around stepped cut at four corners, are proposed. Figure 7 shows the mutual coupling S12, and S21. It can be seen that the mutual coupling S12, S21 of the fabricated antenna are the same, just as is predicted by the simulation of these parameters in the same Figure. From Figure 7, the mutual coupling has dropped below $20 \mathrm{~dB}$.

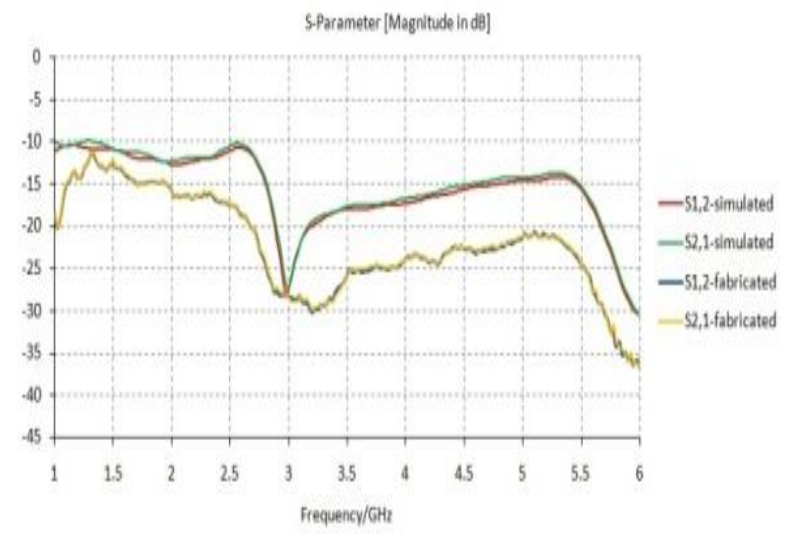

Figure 7. Simulated and measured's coupling coefficient of proposed 2x2 MIMO antenna system. 
Figures 5 and 6 show that the results for the return loss from fabricated design are better than the results from simulation. For instance, Figure 7 shows that the coupling coefficients of the fabricated antenna is below $-20 \mathrm{~dB}$ for the WiMAX bands and under $-15 \mathrm{~dB}$ for LTE and WiFi bands. The fabricated design introduced a new resonant frequency operating at $2.6 \mathrm{GHz}(\mathrm{LTE})$. The bandwidth of all of these frequency bands can be obtained by the reflection coefficient $(\mathrm{S} 11, \mathrm{~S} 22)$ of $-10 \mathrm{~dB}$ or lower. Hence, as opposed to the reference antenna, the results produced by the designed antenna show that it can include more resonant frequencies[5] and is also able to decrease the mutual coupling further compared to the reference antenna.

For the fabricated proposed antenna, the coupling coefficients are below $-20 \mathrm{~dB}$ for the WiMAX (3.5 GHz, $5.2 \mathrm{GHz}$ and $5.5 \mathrm{GHz})$ band and are under $-15 \mathrm{~dB}$ for LTE $(1.8 \mathrm{GHz})$ and WiFi $(2.4 \mathrm{GHz})$ bands.

According to the simulation results as shown in Figure 8, the VSWR of the desired frequency at both ports of the proposed antenna is within the acceptable range $(1<\mathrm{VSWR}<2)$.

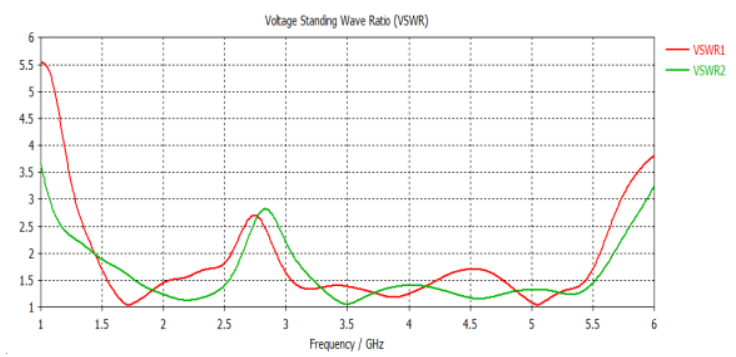

Figure 8. Simulated VSWR over frequency of the proposed 2x2MIMO antenna.

The radiation efficiencies of the proposed 2x2MIMO antenna are shown in Figure 9. This Figure shows that radiation efficiencies of above $70 \%$ have been achieved for all the desired frequencies.

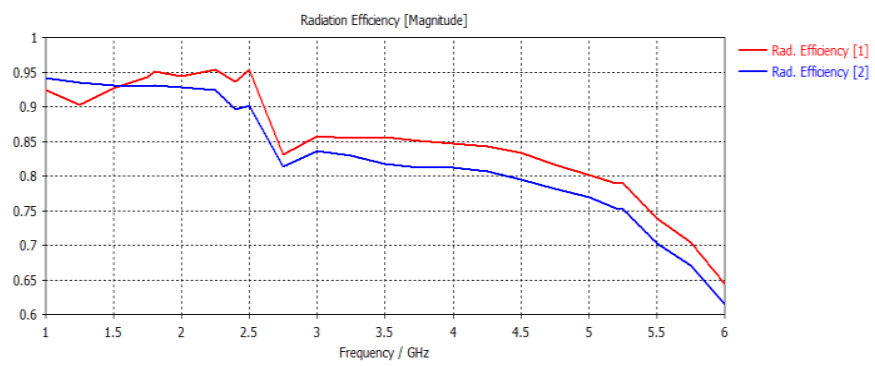

Figure 9. Simulated radiation efficiency of the proposed antenna.

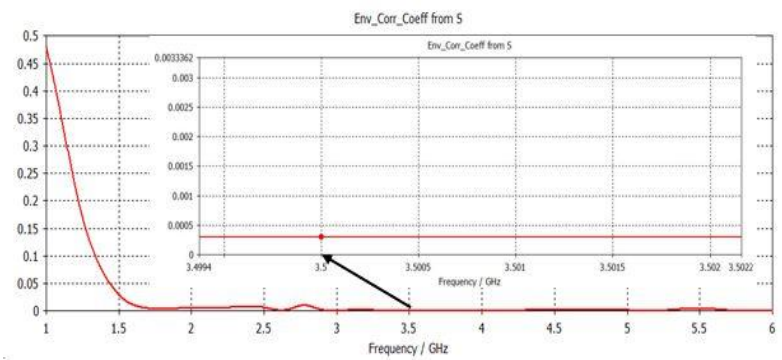

Figure 10. Simulated Envelop Correlation Coefficient of the proposed antenna. 


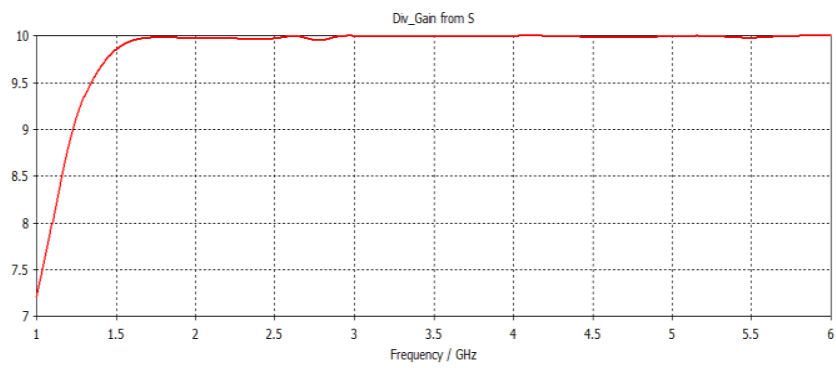

Figure 11. Simulated Diversity gain of the proposed antenna.

The Envelop Correlation Coefficient (ECC) by the proposed 2x2 MIMO antenna is shown in Figure 10. Figure 10 shows that at the desired frequency bands, the ECC is less than 0.007 . However, the ECC is further improved by the improving return loss of the antenna. Hence, it is implied from the result that the proposed antenna system can be used in MIMO applications.

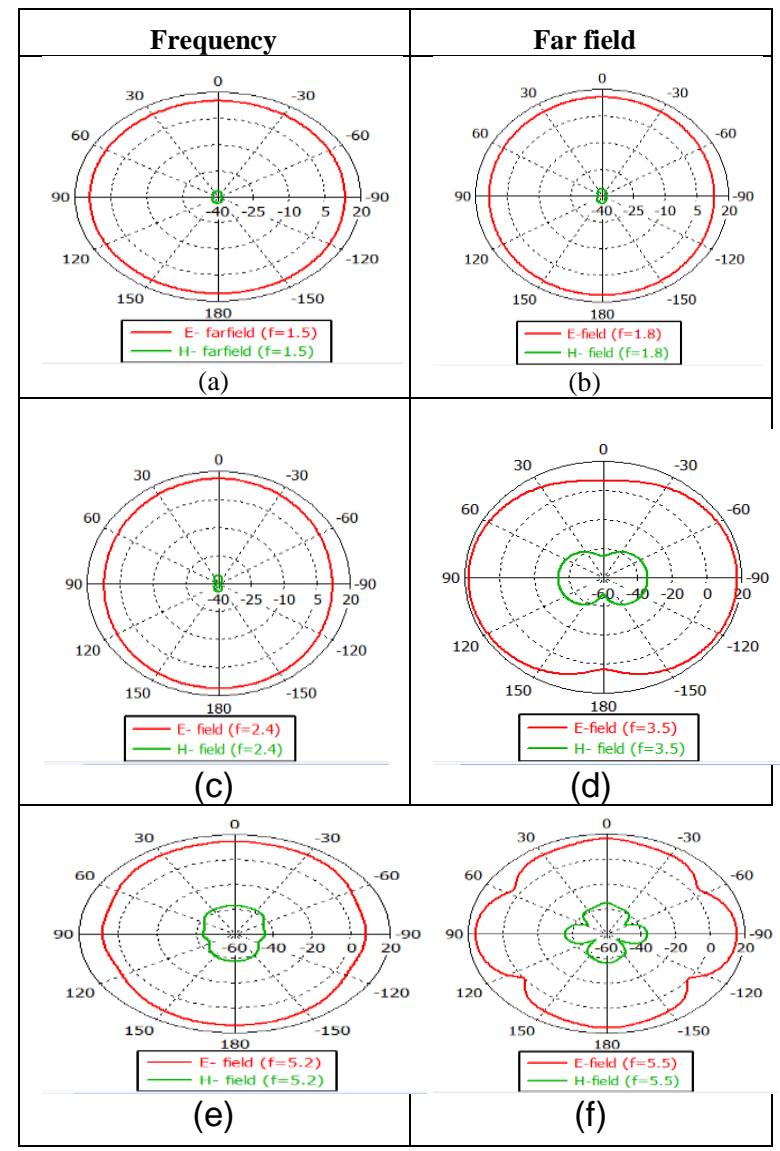

Figure 12. Simulated E-plane and $\mathrm{H}$-plane radiation patterns of $2 \mathrm{X} 2 \mathrm{MIMO}$ proposed antenna (a) $1.5 \mathrm{GHz}$ (b) $1.8 \mathrm{GHz}$ (c) $2.4 \mathrm{GHz}$ (d) $3.5 \mathrm{GHz}$ (e) $5.2 \mathrm{GHz}$ (f) $5.5 \mathrm{GHz}$.

Diversity performance is among the crucial parameters in MIMO antenna. Figure 11 shows that at the desired frequency bands, a Simulated Diversity gain of $9.99 \mathrm{~dB}$ is achieved for the $2 \times 2$ MIMO antenna system. In addition to this, its polar radiation pattern has been simulated in the $x-z$ (H-plane) and $y-z$ (E-plane) at the desired frequencies as shown in Figure 12. The 
desired frequencies are $1.5 \mathrm{GHz}, 1.8 \mathrm{GHz}, 2.4 \mathrm{GHz}, 3.5 \mathrm{GHz}, 5.2 \mathrm{GHz}$ and $5.5 \mathrm{GHz}$. Figure 12 also shows that the proposed system possesses an omni-directional pattern at low frequencies while this radiation pattern changes to Quasi-omni-directional pattern at high frequencies like $5.5 \mathrm{GHz}$.

\section{Conclusion}

In efforts to reduce the mutual coupling effects, and to enhance the efficiency and bandwidth, a multiband $2 \times 2$ MIMO microstrip patch antenna with rings and mid-slot is proposed. The mutual coupling has been reduced using a unique method by applying mid slot between the two ports. In addition to this, more resonant frequencies have been obtained by using four groups of rings positioned around stepped cut at four corners to enhance the bandwidth and efficiency. The designed antenna has been simulated and compared with a reference antenna. The diversity gain of $9.99 \mathrm{~dB}$ and ECC of 0.007 were achieved. Moreover, all desired frequencies achieved radiation efficiencies of $70 \%$ and above. Therefore, the proposed antenna with its small dimensions can be useful for LTE (1.8 GHz), WiFi $(2.4 \mathrm{GHz})$, and WiMax (3.5 GHz, $5.2 \mathrm{GHz}$ and $5.5 \mathrm{GHz}$ ) applications.

\section{References}

[1] Babu KJ, Krishna KSR, Reddy LP. A review on the design of MIMO antennas for upcoming 4G communications. International journal of applied engineering research. 2011; 1(4): 85-93.

[2] Alsaleh AAA, Islam MR, Wan Nordin MA, Al-Askari S. Design and Optimization of Dual Band Microstip Patch Antenna Using Slots Pair. Proceedings of 2016 International Conference on Computer \& Communication Engineering, DOI 10.1109/ICCCE.2016.98, 2016:439-442.

[3] Chourasia S and Changlani DS. Design and Analysis of I-Shaped Microstrip Patch Antenna for Low Frequency. IJIRST -International Journal for Innovative Research in Science and Technology. 2014; 1(6): 320-324.

[4] Adnan NHM, Islam MR and Alam AHMZ. Massive MIMO for Fifth Generation (5G): Opportunities and Challenges. Proceedings of 2016 International Conference on Computer \& Communication Engineering, DOI 10.1109/ICCCE.2016.23, 2016:47-52.

[5] Li Y, Zheng J, Feng Z. Latest Progress in MIMO Antennas Design. INTECH Open Access Publisher. 2012: 1-6.

[6] MoradiKordalivand A, Rahman TA. Broadband modified rectangular microstrip patch antenna using stepped cut at four corners method. Progress In Electromagnetics Research. 2013; 137: 599-619.

[7] Abdulrab WS, Islam MR, Habaebi MH. Design Of Rectangular Microstrip Patch Antenna Using Stepped Cut At Four Corners For Broadband/Multiband Application. ARPN Journal of Engineering and Applied Science. 2015; 10(22): 17265-17269.

[8] Moradikordalivand A, Rahman TA, Fadoul MM. Evolution Process of a Wideband Dual Polarized MIMO antenna System for Wireless Access Point Applications. 7th International Symposium on Telecommunications (IST'2014). 2014: 266-269. 